\title{
SPITZER SPECTROSCOPY OF THE CIRCUMPRIMARY DISK IN THE BINARY BROWN DWARF 2MASS J04414489+2301513*
}

\author{
Lucía Adame ${ }^{1,2}$, Nuria Calvet ${ }^{1}$, K. L. Luhman ${ }^{3,4}$, Paola D’Alessio ${ }^{5}$, Elise Furlan ${ }^{6}$, M. K. McClure $^{1}$, \\ LeE Hartmann $^{1}$, William J. Forrest ${ }^{7}$, and Dan M. Watson ${ }^{2}$ \\ ${ }^{1}$ Department of Astronomy, University of Michigan, Ann Arbor, MI 48109, USA \\ ${ }^{2}$ Instituto de Astronomía, Universidad Nacional Autónoma de México, AP 70-264, 04510 México DF, Mexico; adamel@umich.edu \\ ${ }_{3}$ Department of Astronomy and Astrophysics, The Pennsylvania State University, University Park, PA 16802, USA \\ ${ }^{4}$ Center for Exoplanets and Habitable Worlds, The Pennsylvania State University, University Park, PA 16802, USA \\ ${ }^{5}$ Centro de Radioastronomía y Astrofísica, Universidad Nacional Autónoma de México, Apartado Postal 72-3 (Xangari), 58089 Morelia, Michoacán, Mexico \\ ${ }^{6}$ JPL, Caltech, Mail Stop 264-767, 4800 Oak Grove Dr., Pasadena, CA 91109, USA \\ ${ }^{7}$ Department of Physics and Astronomy, The University of Rochester, Rochester, NY 14627, USA \\ Received 2010 August 10; accepted 2010 November 22; published 2010 December 7
}

\begin{abstract}
Using the Spitzer Infrared Spectrograph, we have performed mid-infrared spectroscopy on the young binary brown dwarf 2MASS J04414489+2301513 (15 AU) in the Taurus star-forming region. The spectrum exhibits excess continuum emission that likely arises from a circumstellar disk around the primary. Silicate emission is not detected in these data, indicating the presence of significant grain growth. This is one of the few brown dwarf disks at such a young age ( $\sim 1 \mathrm{Myr})$ that has been found to lack silicate emission. To quantitatively constrain the properties of the disk, we have compared the spectral energy distribution of 2MASS J04414489+2301513 to the predictions of our vertical structure codes for irradiated accretion disks. Our models suggest that the remaining atmospheric grains of moderately depleted layers may have grown to a size of $\gtrsim 5 \mu \mathrm{m}$. In addition, our model fits indicate an outer radius of $0.2-0.3 \mathrm{AU}$ for the disk. The small size of this circumprimary disk could be due to truncation by the secondary. The absence of an outer disk containing a reservoir of small, primordial grains, combined with a weak turbulent mechanism, may be responsible for the advanced grain growth in this disk.
\end{abstract}

Key words: accretion, accretion disks - binaries: close - brown dwarfs - circumstellar matter - stars: individual (2MASS J04414489+2301513)

\section{INTRODUCTION}

Grain growth, crystallization, and settling occur in primordial circumstellar disks around both stellar and substellar objects. These processes produce several observational signatures. The mid-infrared (IR) silicate emission becomes weaker as the grains in the upper layers grow and settle toward the midplane while crystallization modifies the shape of the band. IR continuum emission is reduced at longer wavelengths with grain growth and settling because the intercepted stellar flux decreases as the disk flattens. In the substellar domain, these hallmarks of dust evolution have been reported previously for disks at ages $\tau \sim 1-10 \mathrm{Myr}$ (Apai et al. 2004, 2005; Scholz et al. 2007; Morrow et al. 2008; Pascucci et al. 2009; Riaz 2009; Riaz et al. 2009). For instance, disks around the youngest brown dwarfs exhibit evidence of small, crystalline silicate grains (Apai et al. 2005; Riaz 2009) while older brown dwarf disks have weaker silicate emission that indicates the presence of large grains (Scholz et al. 2007; Morrow et al. 2008; Riaz et al. 2009). The evolution of grains in the inner annuli of these disks, e.g., the zone where the $10 \mu \mathrm{m}$ silicate band emerges (within $10^{-3}$ to $0.1 \mathrm{AU}$; Kessler-Silacci et al. 2007), appears to occur more rapidly than in disks around solar-mass stars. One possible explanation for this phenomena is that the low levels of turbulence for brown dwarf disks produce lower mass accretion rates and reduce the replenishment of the inner disk with primordial grains from outer annuli (Sicilia-Aguilar et al. 2007). Also, the colli-

\footnotetext{
* Based on observations made with the Spitzer Space Telescope, which is operated by the Jet Propulsion Laboratory, California Institute of Technology
} under a contract with NASA. sional rate between large size grains would be small, preventing the fragmentation to small size grains.

During a mid-IR spectroscopic survey of young brown dwarfs with the Spitzer Space Telescope (Werner et al. 2004), we have identified a brown dwarf disk that shows evidence of advanced grain evolution at a relatively young age of $\sim 1$ Myr. This brown dwarf, 2MASS J04414489+2301513 (Luhman 2006, henceforth 2M J04414489), lies in the Taurus star-forming region and was recently shown to harbor a companion with a projected separation of 0 '. $105(\sim 15 \mathrm{AU})$ and a mass of 5-10 $M_{\text {Jup }}$ (Todorov et al. 2010). In this Letter, we use our mid-IR spectrum of this system and our models of settled, irradiated accretion disks to constrain the properties of the circumstellar disk that resides around the primary.

\section{OBSERVATIONS}

We observed 2M J04414489 with the Spitzer Infrared Spectrograph (IRS; Houck et al. 2004) on 2009 April 20 as a part of the Guaranteed Time Observations of the IRS instrument team. We collected data with both of the low-resolution IRS modules, Short-Low (SL) and Long-Low (LL), which cover 5.3-14 and 14-40 $\mu \mathrm{m}$, respectively, with a resolution of $\lambda / \Delta \lambda \sim 90$. We used the basic calibrated data produced by version $S 18.7$ of the Spitzer Science Center pipeline. Before extraction of the spectrum, bad and "rogue" pixels were fixed by interpolating adjacent, good pixels in the dispersion direction. 2M J04414489 was then extracted using the optimal extraction tool in SMART (Higdon et al. 2004; Lebouteiller et al. 2010). We chose the default calibration, which is based on a relative spectral response function derived from three calibrator stars. The young low-mass star 2MASS J04414565+2301580 is 12 ".4 from 2M 
J04414489 and fell within the slit during the LL observations. We performed optimal extraction at the nominal nod position of 2M J04414489 to extract its spectrum, which should result in minimal contamination from 2MASS J04414565+2301580. Indeed, the resulting flux levels of the data at SL and LL match at $14 \mu \mathrm{m}$ without applying any scaling factors. The IRS spectrum includes the flux of both 2M J04414565 A and B, but it should be dominated by the former, as explained in Section 3.1.

\section{ANALYSIS}

\subsection{Disk Emission}

We show the spectral energy distribution (SED) of $2 \mathrm{M}$ J04414489 in Figure 1, which was constructed with our IRS data, 0.8-2.5 $\mu \mathrm{m}$ spectroscopy from Luhman (2006), and 3-8 $\mu \mathrm{m}$ photometry from Luhman et al. (2010). We have included in Figure 1 the SED for a diskless young brown dwarf near the same spectral type as 2M J04414489 (2MASSW J1139511-315921; Morrow et al. 2008). Relative to the stellar photosphere of the latter object, the SED of 2M J04414489 exhibits significant excess emission that indicates the presence of a dusty, optically thick disk, which is consistent with earlier results based on the mid-IR photometry (Luhman et al. 2010). The disk is probably located around the primary because a disk around the secondary would be too faint to account for the observed excess emission based on the flux ratio at near-IR wavelengths ( $\Delta K_{s}=1.54$; Todorov et al. 2010) and the typical near-IR to mid-IR colors of brown dwarfs with disks (Luhman et al. 2010). A circumbinary disk is not plausible since its inner hole would be too large for the disk to produce significant midIR emission. Finally, the mid-IR excess emission cannot be due to the photosphere of the secondary since its IR colors should be similar to those of the primary's photosphere. For instance, given the spectral type of M8.5 for the primary and an expected type of M9.5-L0 for the secondary (Luhman 2006; Todorov et al. 2010), their photospheres should have colors near $K_{s}-[4.5]=0.71$ and 0.83, respectively (Luhman et al. 2010). Combining those colors with the flux ratio at $K_{s}$ suggests that the secondary's photosphere produces an excess of only $\sim 0.02$ mag in $K_{s}-$ [4.5] for the system relative to the photospheric colors of the primary.

\subsection{Model Parameters}

The optical spectrum of 2M J04414489 exhibits $\mathrm{H} \alpha$ emission (Luhman 2006), indicating the presence of ongoing mass accretion. Therefore, we have modeled the mid-IR excess emission from 2M J04414489 in terms of emission from a settled, irradiated accretion disk following the procedures of D'Alessio et al. (1998, 2005, 2006) adapted for substellar objects (Adame 2010). For the stellar photosphere, we adopt an effective temperature of $2555 \mathrm{~K}$, which corresponds to its M8.5 spectral type (Luhman et al. 2003; Luhman 2006). We also adopt a luminosity of $0.004 L_{\odot}$ based on its $J$ magnitude and a mass of $0.025 M_{\odot}$ based on theoretical evolutionary models (Chabrier et al. 2000). In our models, we solve the vertical structure of the disk while assuming a constant turbulent-viscosity parameter $\alpha$ and a uniform mass accretion rate, $\dot{M}$, through the disk. For most of the brown dwarfs in Taurus, assuming $\alpha \geqslant 10^{-3}$ is suitable (Adame 2010), although we allow $\alpha$ to range from $10^{-5}$ to 0.01 for our models of $2 \mathrm{M} \mathrm{J04414489.} \mathrm{In} \mathrm{each} \mathrm{annulus,}$ the mass surface density depend on the ratio $\dot{M} / \alpha$. We compute models with $\dot{M}=10^{-12}, 10^{-11}$, and $10^{-10} M_{\odot} \mathrm{yr}^{-1}$, which are typical values measured for young substellar objects (Natta

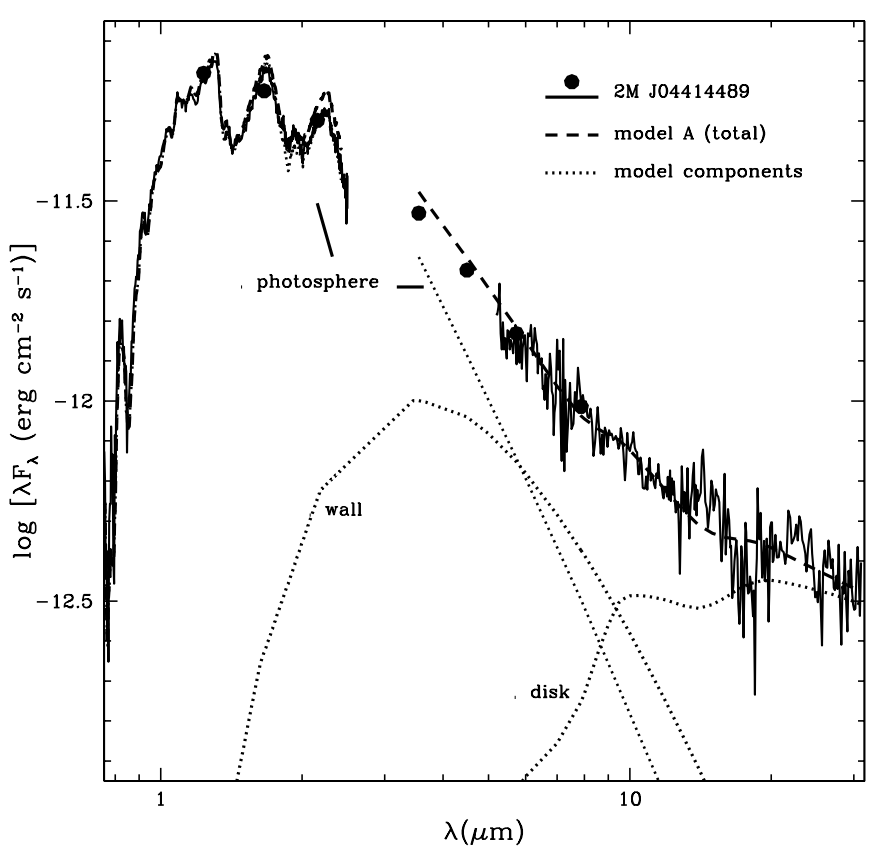

Figure 1. SED of the young brown dwarf 2M J04414489 compared to a model of its circumstellar disk (dotted and dashed lines, model A from Table 1). We have adopted the SED of 2MASSW J1139511-315921 (Morrow et al. 2008) to represent the stellar photosphere and have scaled it to the 1-2 $\mu \mathrm{m}$ flux of $2 \mathrm{M}$ J04414489. For $\lambda \leqslant 2.5 \mu \mathrm{m}$, the stellar photosphere overlaps almost precisely with the photosphere of 2M J04414489.

et al. 2004; Muzerolle et al. 2005; Mohanty et al. 2005; Herczeg \& Hillenbrand 2008; Herczeg et al. 2009).

The adopted dust model is composed of segregated spheres of astronomical silicates and graphite with abundances and optical constants from Draine \& Lee (1984) and Weingartner \& Draine (2001) and a size distribution of $n(a) \sim a^{-p}$ (Mathis et al. 1977 ), where $p=3.5$. To account for grain growth, we have varied the maximum grain size in the upper layers and the inner wall, adopting values of $a_{\max }=0.25,1,5,10,100$, and $1000 \mu \mathrm{m}$. Meanwhile, we assume $a_{\max }=1 \mathrm{~mm}$ for the dust population near the midplane. As grains grow and settle toward the midplane, the atmosphere is depleted of small grains. The model of D'Alessio et al. (2006) quantifies this depletion through the parameter $\epsilon$, which is the dust-to-gas mass ratio of the atmosphere relative to the standard ratio (with $\zeta_{\text {sil }}=0.004$ and $\zeta_{\text {graph }}=0.0025$; Draine \& Lee 1984). We have considered $\epsilon=0.001,0.01$, and 0.1 in our models.

We also calculate the structure and SED of the inner wall, which is heated by radiation from the stellar photosphere (D'Alessio et al. 2005). The wall is located at radius $R_{\text {in }}$, which can be the dust destruction radius (assuming a sublimation temperature of $\sim 1400 \mathrm{~K}$ ) or the magnetospheric radius, whichever is larger. We explore maximum dust temperatures of $\sim 1400$ $1000 \mathrm{~K}$ (in steps of $100 \mathrm{~K}$ ), corresponding to an inner dust gap with $R_{\text {in }} \sim 3-6 R_{*}$. The outer disk radius $R_{d}$ may be set by interaction with the secondary (Papaloizou \& Pringle 1977; Artymowicz \& Lubow 1994). Assuming a mass ratio of the secondary to the primary of $\sim 0.3$, a projected semimajor axis of $\sim 15$ AU (Todorov et al. 2010), and a mean Reynolds number of $10^{4}-10^{5}$ (see Artymowicz \& Lubow 1994), the truncation radius is predicted to be $3 \mathrm{AU} \lesssim R_{\text {truncation }} \lesssim 7 \mathrm{AU}$ for eccentricities between $e=0$ and $e=0.5-0.6$ (a large circumprimary radius corresponds to a small eccentricity). Therefore, we fixed $R_{d}$ to an initial value of $5 \mathrm{AU}$; for promising models, we have selected values of $R_{d}$ that range from 0.1 to $5 \mathrm{AU}$. Finally, we 


\begin{tabular}{lccccccc}
\multicolumn{7}{c}{$\begin{array}{c}\text { Table 1 } \\
\text { Models }\end{array}$} \\
\hline \hline Model $^{\mathrm{b}}$ & $\epsilon$ & $a_{\text {max }}$ & $z_{\text {wall }}$ & $R_{d}$ & $M_{\text {dust }}$ & $\chi_{r}^{2}$ & $t_{\text {RM }}$ \\
& & $(\mu \mathrm{m})$ & $\left(H_{p}\right)$ & $(\mathrm{AU})$ & $\left(M_{\text {Moon }}\right)$ & & $\left(10^{6}\right.$ yr $)$ \\
\hline A & 0.1 & 10 & 3.7 & 0.2 & 3.2 & 3.7 & 1.2 \\
B & 0.1 & 5 & 3.6 & 0.2 & 3.2 & 3.8 & 1.2 \\
C & 0.1 & 100 & 3.9 & 0.3 & 5.2 & 3.9 & 1.8 \\
D & 0.01 & 5 & 3.8 & 0.3 & 5.7 & 3.9 & 1.9 \\
\hline
\end{tabular}

\section{Notes.}

a The listed parameters are the dust-depletion parameter $\epsilon$, the maximum grain radius of the dust population in the disk atmosphere $a_{\max }$, the height of the wall $z_{\text {wall }}$, the outer disk radius $R_{d}$, the total dust content of the disk $M_{\text {dust }}$, the reduced $-\chi^{2}$ value of the model, and the characteristic timescale for the radial mixing, evaluated at $R_{d}, t_{\mathrm{RM}}$.

${ }^{\mathrm{b}} \alpha=10^{-5}, \dot{M}=10^{-11} M_{\odot} \mathrm{yr}^{-1}, i=20^{\circ}$, and $R_{\text {in }}=6 R_{*}$ for all models.

adopt inclination angles of $i=10^{\circ}, 20^{\circ}$, and $60^{\circ}$. The disk SED library consists of some 700 models.

\subsection{Model Fits}

We proceed to identify the combinations of disk parameters that can reproduce the SED of 2M J04414489. We perform a reduced- $\chi^{2}$ goodness-of-fit test between each model and the IRS data (e.g., van Boekel et al. 2005), where $\chi_{r}^{2} \sim 1$ indicates a good match. We find that models with $i=20^{\circ}, 10^{-11}$ $M_{\odot} \mathrm{yr}^{-1} \leqslant \dot{M} \leqslant 10^{-10} M_{\odot} \mathrm{yr}^{-1}$, and a wall temperature of $\sim 1000 \mathrm{~K}$ match the SED at $\lambda<10 \mu \mathrm{m}$ better than other values of these parameters. The radius of this inner wall is $R_{\text {in }} \approx 6 R_{*} \approx 0.009 \mathrm{AU}$. We allow the wall height $z_{\text {wall }}$ to vary from $3.4 H_{p}$ to $4.1 H_{p}$, where $H_{p}$ is the gas scale height at $R_{\text {in }}$. The models with these values of $i, M$, and $R_{\text {in }}$ that produce $\chi_{r}^{2}<4$ are 21. An important constrain for our model SED selection is the value of the mass accretion rate. From the $\dot{M}-M_{*}$ relationship of Herczeg \& Hillenbrand (2008), the mass accretion rate onto this low-mass brown dwarf would be $\log \dot{M} \approx-10.7 \pm 0.6$, thus models with $10^{-10} M_{\odot} \mathrm{yr}^{-1}$ are in the higher end of the expected mass accretion rate. On the other hand, the equivalent width of the $\mathrm{H} \alpha$ emission line of $2 \mathrm{M}$ J04414489 (Luhman 2006) is $100 \AA$, which corresponds to a $10 \%$ width of $\mathrm{H} \alpha$ of $\sim 220 \mathrm{~km} \mathrm{~s}^{-1}$ (Muzerolle et al. 2005), and to a mass accretion rate of $\lesssim 10^{-11} M_{\odot} \mathrm{yr}^{-1}$ (Natta et al. 2004). Hence, we discard the high mass accretion models, and select the remaining four models as our best-fit models, which are listed in Table 1.

The SED predicted by model A, which has the lowest value of $\chi_{r}^{2}$, is compared to the observed SED in Figure 1. The variations in the disk parameters among the best-fit models in Table 1 are qualitatively explained by the fact that the mid-IR emission is weaker for smaller $\epsilon$ and $\dot{M}$ and larger $a_{\max }$ and $\alpha$, causing a reduced optical depth. For instance, the grain growth of model $\mathrm{C}$ and the depletion of model $\mathrm{D}$ must be balanced by increasing the dust mass content in order for the models to produce sufficient emission to match the data. Meanwhile, models with $a_{\max }=1 \mathrm{~mm}$ and most of the models with $10^{-12} M_{\odot} \mathrm{yr}^{-1} \leqslant \dot{M} \leqslant 10^{-11} M_{\odot} \mathrm{yr}^{-1}$ produced emission that was too weak to reproduce the data $\left(\chi_{r}^{2} \gg 4\right)$.

We compare the silicate emission predicted by our best-fit models to the IRS data for 2M J04414489 in Figure 2. We normalize the IRS spectrum and the models with a continuum interpolated from a third-order polynomial constructed by fluxes between $\sim 5.2$ and $\sim 7.9 \mu \mathrm{m}$ and between $\sim 13$ and $16 \mu \mathrm{m}$ (e.g., Furlan et al. 2006). The strength of the silicate feature relative

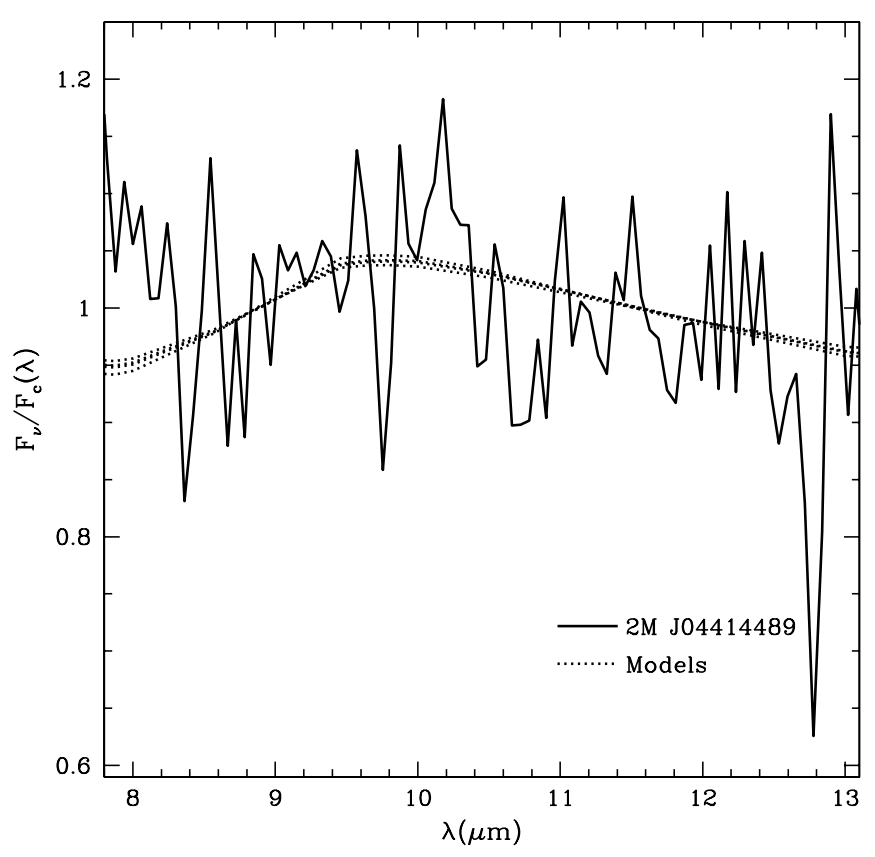

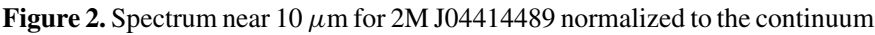
surrounding the wavelengths of the silicate feature. For comparison, we show the normalized silicate emission produced by the models in Table 1 . The order of the models from strongest to weakest emission peak is B, A, C, and D.

to the continuum is obtained by subtracting the continuum to the feature, integrating this residual flux, and normalize it to the integrated continuum flux between 8 and $13 \mu \mathrm{m}$ (Furlan et al. 2006). The strength of the observed feature is 1.03 , whereas the strengths of our models features range from 1.024 to 1.032 . Thus, the observed and modeled silicate features are similar, but also, they are extremely weak. The absence of detectable silicate emission in the data indicates that grains in the disk atmosphere have grown to sizes of $5 \mu \mathrm{m} \lesssim a_{\max }<1000 \mu \mathrm{m}$, which are larger than the typical interstellar medium grains.

The dust masses of our final set of disk models are listed in Table 1, using $\zeta_{\text {std }}=0.0065$ (Draine \& Lee 1984). The masses of the models range from $\sim 3$ to $\sim 5 M_{\text {Moon }}$, which are more than an order of magnitude lower than the values estimated from millimeter measurements of other brown dwarf disks in Taurus (Scholz et al. 2006). This result is not surprising given that close binary stars exhibit low disk masses compared to single stars and wide binaries (Jensen et al. 1994).

\subsection{Size of the Mid-IR Emission Region}

The mid-IR emission from an isolated disk irradiated by a M8.5 brown dwarf typically forms within $R_{\mathrm{MIR}} \lesssim 5 \mathrm{AU}$. The exact size of this region depends on the size of the grains (Kessler-Silacci et al. 2007), but also on the dust mass surface density through the variation of $\epsilon, \dot{M}$, or $\alpha$. We find that decreasing $\alpha$ or increasing $\dot{M}$ or $\epsilon$ result in a wider emitting region since those variations increase the irradiation surface and the impinging irradiation flux at any given radius. On the other hand, decreasing $a_{\max }$ will increase the optical depth to the incident radiation at the upper layers, which in turn extends vertically outward the irradiation surface, allowing more distant annuli to contribute to the emission.

If the outer radius of the disk is smaller than the nominal radius of the mid-IR emitting region, then the mid-IR SED is sensitive to the value of the outer radius. For instance, the radii for all of the models in Table 1 are constrained to 


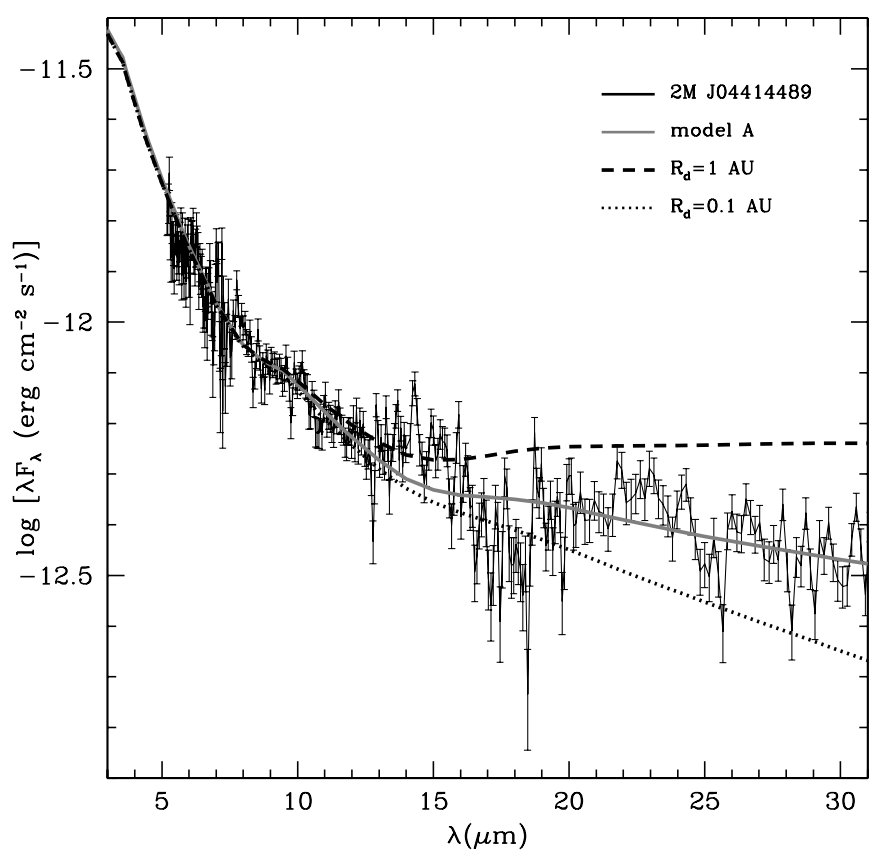

Figure 3. SED of the young brown dwarf $2 \mathrm{M}$ J04414489 compared to model $\mathrm{A}\left(R_{d}=0.2 \mathrm{AU}\right)$ and two models that have the same parameters as model $\mathrm{A}$ except for different values of $R_{d}$.

be $0.2 \mathrm{AU} \lesssim R_{d} \lesssim 0.3 \mathrm{AU}$ by the mid-IR SED of $2 \mathrm{M}$ $\mathrm{J} 04414489$, which is smaller than the expected radius given by the binary tidal interaction $\left(R_{\text {truncation }} \sim 3-7 \mathrm{AU}\right)$. However, if the eccentricity of the binary is high enough, the periastron would be close to $1 \mathrm{AU}$ if a semimajor axis of $15 \mathrm{AU}$ is still assumed. The dependence of the predicted SED on the outer radius is illustrated in Figure 3 for model A. Although the disk

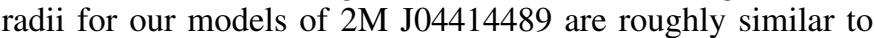
that derived for the circumprimary disk of SR 20 (0.39 AU, for a G7 primary and a binary projected separation of 5.3-9.9 AU; McClure et al. 2008), the former has a redder SED (i.e., less affected by truncation) because the mid-IR emitting region of a brown dwarf is much smaller than that of a star.

\section{DISCUSSION}

We find that the dust in the disk around 2M J04414489 is relatively evolved for the age of the system $(\sim 1 \mathrm{Myr})$. Based on the slope of its SED and the absence of silicate emission, most of the initial atmospheric grains have settled toward the midplane $(0.01 \leqslant \epsilon \leqslant 0.1)$ and the remaining grains may have experienced significant growth $\left(5 \mu \mathrm{m} \lesssim a_{\max }<\right.$ $1000 \mu \mathrm{m})$, representing one of $\sim 2$ brown dwarf disks in Taurus that show evidence of accelerated dust evolution (see KPNO $6^{8}$; Riaz 2009). This degree of dust evolution has been observed primarily for brown dwarfs at ages of 5-10 Myr (Scholz et al. 2007; Morrow et al. 2008).

Some mechanisms to explain grain growth and settling in disks around either single or multiple late-type M-dwarfs have been put forward. Meeus et al. (2003) suggested that binary interaction may accelerate the dust evolution through an enhanced vertical stirring of grains from the midplane. However, Pascucci et al. (2008) and E. Furlan et al. (2011, in preparation) found no significant differences between the

\footnotetext{
8 For the other objects reported by Riaz (2009), silicate emission is detected at weak levels in the same spectra that were re-reduced (E. Furlan et al. 2011, in preparation).
}

silicate feature for early-type single and binary star systems in Taurus, revealing that binary interaction may not affect the inner disk region, although the outermost region is indeed influenced. The interaction truncates the disk to a given radius (Papaloizou \& Pringle 1977; Artymowicz \& Lubow 1994), decreasing the emission from a certain wavelength range. For instance, the submillimeter and millimeter emission from the circumprimary disk is diminished for projected binary separations of $\sim 50$ 100 AU (Jensen et al. 1994, 1996), while for smaller separations ( $\lesssim 50 \mathrm{AU})$, the mid-IR emission of the outer disk decreases with respect to that of the innermost region (McClure et al. 2010). The mid-IR emission of J04414489 is the result of this truncation, whereas the fluxes near $\sim 10 \mu \mathrm{m}$ are unlikely influenced by the binary interaction. Instead, the inner region may be probably affected by the turbulence as most of the eddie dissipation occurs within those annuli ( $\lesssim 0.1 \mathrm{AU})$, which represents $\sim 30 \%-50 \%$ of the total radial extension of the disk.

Grain growth and settling should be efficient in regions close to the star, where temperatures and densities are larger, and the turbulent movements are higher (Beckwith et al. 2000; Dullemond \& Dominik 2004; Nomura \& Nakagawa 2006, references therein). For this weakly turbulent disk $\left(\alpha=10^{-5}\right)$, the coagulation rate may be slowed down, but also the relative velocities of the grains, thus preventing their fragmentation. The smaller velocity gradients can work to preserve the large grains once formed. A radial mixing mechanism may exist that moves evolved grains outward and replenishes the inner disk with primordial dust (e.g., turbulent diffusion, convection, gravitational torques, among others; see Gail 2001; BockeléeMorvan et al. 2002; Boss 2004). The primordial dust reservoir is limited by the outer radius of a disk. If a disk is outwardly truncated to a small enough radius (e.g., $R_{d}<R_{\mathrm{MIR}}$ ), the outermost region will contain evolved grains, which return to the inner region to be processed further. The characteristic timescale for this radial mixing is approximately the viscous timescale (Bockelée-Morvan et al. 2002), which for an $\alpha$ disk, $t_{\mathrm{RM}}=R_{0}^{2} \Omega_{K}\left(R_{0}\right) / 3 \alpha c_{s}^{2}\left(T_{c}\right)$. Here, $c_{s}\left(T_{c}\right)$ is the sound speed at the midplane and $\Omega_{K}$ is the Keplerian angular speed, both evaluated at $R_{0}$ (the initial outer radius of the disk). We evaluate $t_{\mathrm{RM}}$ at the outer radii listed in Table 1, assuming a central temperature at the midplane of $\sim 60-80 \mathrm{~K}$. The mixing timescale derived is $\sim 1-2 \mathrm{Myr}$, which is comparable to the age of $2 \mathrm{M} \mathrm{J04414489.} \mathrm{The} \mathrm{radial} \mathrm{mixing} \mathrm{may} \mathrm{be} \mathrm{at} \mathrm{an} \mathrm{earliest} \mathrm{stage,}$ but its influence over the dust processing cannot be neglected. Therefore, low levels of turbulence combined with a highly truncated disk could explain the deficit of primordial dust that we observe in the disk of $2 \mathrm{M} \mathrm{J04414489.}$

\section{CONCLUSIONS}

We have presented a mid-IR spectrum of the binary brown dwarf 2M J04414489 in the Taurus star-forming region. The spectrum exhibits excess emission that we attribute to a circumprimary disk, but silicate emission at $10 \mu \mathrm{m}$ is not present, indicating that significant grain growth has occurred, leading to a disk on which small grains no longer exist $\left(5 \mu \mathrm{m} \lesssim a_{\max }<\right.$ $1000 \mu \mathrm{m})$. This is one of $\sim 2$ brown dwarf disks at such a young age $(\sim 1 \mathrm{Myr})$ that have been found to lack silicate emission. Our models of the SED of 2M J04414489 suggest that the outer radius of its disk is rather small $\left(R_{d} \sim 0.2-0.3 \mathrm{AU}\right)$, which may reflect truncation by the binary companion. The absence of an outer disk with a reservoir of primordial grains, combined with weak turbulence, could explain the advanced grain growth in the inner disk of $2 \mathrm{M} \mathrm{J04414489.}$ 
We thank the anonymous referee for his/her valuable comments. We acknowledge support from grant IN112009 from PAPIIT-DGAPA UNAM (P.D. and L.A.), grant NNX08AH94G from NASA (N.C. and L.A.), and grant AST-0544588 from the National Science Foundation (K.L.). Part of the numerical calculations were performed on the cluster at CRyA-UNAM, acquired through CONACyT grant 36571-E to Enrique VázquezSemadeni. The Center for Exoplanets and Habitable Worlds is supported by the Pennsylvania State University, the Eberly College of Science, and the Pennsylvania Space Grant Consortium.

\section{REFERENCES}

Adame, L. 2010, PhD thesis, Universidad Nacional Autónoma de México Apai, D., Pascucci, I., Bouwman, J., Natta, A., Henning, T., \& Dullemond, C. P. 2005, Science, 310, 834

Apai, D., Pascucci, I., Sterzik, M. F., van der Bliek, N., Bouwman, J., Dullemond, C. P., \& Henning, T. 2004, A\&A, 426, L53

Artymowicz, P., \& Lubow, S. H. 1994, ApJ, 421, 651

Beckwith, S. V. W., Henning, T., \& Nakagawa, Y. 2000, in Protostars and Planets IV, ed. V. Mannings, A. P. Boss, \& S. S. Russell (Tucson, AZ: Univ. Arizona Press), 533

Bockelée-Morvan, D., Gautier, D., Hersant, F., Huré, J.-M., \& Robert, F. 2002, A\&A, 384, 1107

Boss, A. P. 2004, ApJ, 616, 1265

Chabrier, G., Baraffe, I., Allard, F., \& Hauschildt, P. 2000, ApJ, 542, 464

D’Alessio, P., Calvet, N., Hartmann, L., Franco-Hernández, R., \& Servín, H. 2006, ApJ, 638, 314

D’Alessio, P., Cantó, J., Calvet, N., \& Lizano, S. 1998, ApJ, 500, 411

D’Alessio, P., et al. 2005, ApJ, 621, 461

Draine, B. T., \& Lee, H. M. 1984, ApJ, 285, 89

Dullemond, C. P., \& Dominik, C. 2004, A\&A, 421, 1075

Furlan, E., et al. 2006, ApJS, 165, 568

Gail, H.-P. 2001, A\&A, 378, 192

Herczeg, G. J., Cruz, K. L., \& Hillenbrand, L. A. 2009, ApJ, 696, 1589

Herczeg, G. J., \& Hillenbrand, L. A. 2008, ApJ, 681, 594

Higdon, S. J. U., et al. 2004, PASP, 116, 975
Houck, J. R., et al. 2004, ApJS, 154, 18

Jensen, E. L. N., Mathieu, R. D., \& Fuller, G. A. 1994, ApJ, 429, L29

Jensen, E. L. N., Mathieu, R. D., \& Fuller, G. A. 1996, ApJ, 458, 312

Kessler-Silacci, J. E., et al. 2007, ApJ, 659, 680

Lebouteiller, V., Bernard-Salas, J., Sloan, G. C., \& Barry, D. J. 2010, PASP, 122,231

Luhman, K. L. 2006, ApJ, 645, 676

Luhman, K. L., Allen, P. R., Espaillat, C., Hartmann, L., \& Calvet, N. 2010, ApJS, 186, 111

Luhman, K. L., Stauffer, J. R., Muench, A. A., Rieke, G. H., Lada, E. A., Bouvier, J., \& Lada, C. J. 2003, ApJ, 593, 1093

Mathis, J. S., Rumpl, W., \& Nordsieck, K. H. 1977, ApJ, 217, 425

McClure, M. K., et al. 2008, ApJ, 683, L187

McClure, M. K., et al. 2010, ApJS, 188, 75

Meeus, G., Sterzik, M., Bouwman, J., \& Natta, A. 2003, A\&A, 409, L25

Mohanty, S., Jayawardhana, R., \& Basri, G. 2005, ApJ, 626, 498

Morrow, A. L., et al. 2008, ApJ, 676, L143

Muzerolle, J., Luhman, K. L., Briceño, C., Hartmann, L., \& Calvet, N. 2005, ApJ, 625, 906

Natta, A., Testi, L., Muzerolle, J., Randich, S., Comerón, F., \& Persi, P. 2004, A\&A, 424, 603

Nomura, H., \& Nakagawa, Y. 2006, ApJ, 640, 1099

Papaloizou, J., \& Pringle, J. E. 1977, MNRAS, 181, 441

Pascucci, I., Apai, D., Hardegree-Ullman, E. E., Kim, J. S., Meyer, M. R., \& Bouwman, J. 2008, ApJ, 673, 477

Pascucci, I., Apai, D., Luhman, K., Henning, T., Bouwman, J., Meyer, M. R., Lahuis, F., \& Natta, A. 2009, ApJ, 696, 143

Riaz, B. 2009, ApJ, 701, 571

Riaz, B., Lodieu, N., \& Gizis, J. E. 2009, ApJ, 705, 1173

Scholz, A., Jayawardhana, R., \& Wood, K. 2006, ApJ, 645, 1498

Scholz, A., Jayawardhana, R., Wood, K., Meeus, G., Stelzer, B., Walker, C., \& O’Sullivan, M. 2007, ApJ, 660, 1517

Sicilia-Aguilar, A., Hartmann, L. W., Watson, D., Bohac, C., Henning, T., \& Bouwman, J. 2007, ApJ, 659, 1637

Todorov, K., Luhman, K. L., \& McLeod, K. K. 2010, ApJ, 714, L84

van Boekel, R., Min, M., Waters, L. B. F. M., de Koter, A., Dominik, C., van den Ancker, M. E., \& Bouwman, J. 2005, A\&A, 437, 189

Weingartner, J. C., \& Draine, B. T. 2001, ApJ, 548, 296

Werner, M. W., et al. 2004, ApJS, 154, 1 